\title{
Corrigendum: Critical fictive temperature for plasticity in metallic glasses
}

\author{
Golden Kumar, Pascal Neibecker, Yan Hui Liu \& Jan Schroers
}

Nature Communications 4:1536 doi: 10.1038/ncomms2546 (2013); Published 26 Feb 2013; Updated 17 Oct 2013

The funding for this Article was not fully acknowledged. The Acknowledgements should have read:

This work was funded by DOE, Office of Basics Energy Sciences through DE SC 0004889, and by the National Science Foundation through MRSEC DMR-1119826. We thank Sindee Simon and Dan Miracle for useful discussions. 